\title{
Energy-Delay Aware Restricted Access Window with Novel Retransmission for IEEE 802.11ah Networks
}

\author{
Yanru Wang*, Kok Keong Chai*, Yue Chen*, John Schormans* and Jonathan Loo ${ }^{\dagger}$ \\ ${ }^{*}$ School of Electronic Engineering and Computer Science, Queen Mary University of London, London, UK \\ Email: \{yanru.wang, michael.chai, yue.chen, j.schormans\}@qmul.ac.uk \\ ${ }^{\dagger}$ Department of Computer Communications, School of Sciences and Technology, Middlesex University, London, UK \\ Email: J.Loo@mdx.ac.uk
}

\begin{abstract}
Restricted Access Window (RAW) has been introduced to IEEE 802.11ah MAC layer to decrease collision probability. However, the inappropriate application of RAW duration for diverse groups of devices would increase uplink energy consumption, delay and lower down the data rate. In this paper, we study a RAW optimization problem with a novel retransmission scheme that utilizes the next empty slot for retransmission in the uplink. The problem is formulated based on overall energy efficiency and delay of each RAW by applying probability theory and Markov Chain. To jointly optimize energy efficiency and delay, an energy-delay aware window control algorithm is proposed to adapt RAW size by estimating the number of time slots and internal slot duration in one RAW for different groups. The optimal solution is derived by applying Gradient Descent approach. Simulation results show that our proposed algorithm improves up to $113.3 \%$ energy efficiency and reduces $53.4 \%$ delay compared to the existing RAW.

Index Terms-IEEE 802.11ah, MAC, RAW, uplink energy efficiency, uplink delay, retransmission
\end{abstract}

\section{INTRODUCTION}

One of the latest wireless communication technologies that been proposed for Internet-of-Things (IoT) applications is the Low Power Wi-Fi [1]. IEEE 802.11ah Wireless LAN standard group has put forward IEEE 802.11ah to support Low Power Wi-Fi (called "Wi-Fi HaLow") [2] [3]. IEEE 802.11ah operates at sub-1 GHz and it can support up to 6000 devices within a network with transmission range up to $1 \mathrm{~km}$ at the rate of more than $100 \mathrm{kbps}$ [4]. Restricted Access Window (RAW) is a new MAC layer feature that used in Low Power Wi-Fi to decrease collision. It limits a set of devices that can access the channel at any time and spreads their attempts over a long period of time [2] [5]. RAW consists of multiple equal time slots, where each slot is selected by devices or assigned to a group of devices for transmission [6] [7]. Devices are in wake-up mode only when turning to their RAW, otherwise would be in doze mode.

There is no standard definition for RAW duration, which affects the communication energy efficiency and delay. For one RAW with long duration, the devices involved should be in active mode for a longer period, leading to expending idle wake-up energy and delay. On the contrary, the collision probability would be high if a large number of devices access through RAW with limited time slots, resulting in high energy consumption and delay. Adaptive internal time slot duration can ensure sufficient time to access and transmit, but also avoid needless idle time. And RAW has an effect on overhead information to inform scheduling information. Meanwhile, since the device selects time slots randomly in uplink communication, there are some empty slots that no device to choose, while some slots are selected by mutiple devices, which will result in collision. Retransmission mechanism for collised devices is a way to reuse the empty slots. Hypothetically, an adaptive RAW control scheme to optimize energy efficiency and delay is required in IEEE 802.11ah based IoT networks

The current work to improve energy efficiency mainly focuses on improving successful transmission probability and reducing collision. In [7], a new medium access control enhancement algorithm was proposed to determine optimal size of RAW by applying Maximum Likelihood (ML) estimation method to estimate the number devices for uplink access. However, it only involves successful transmission probability without consideration of energy. In [8], the authors introduced Successive Interference Cancellation to improve the throughput in limited time but this could result in more collisions. In [9] and [10], new algorithms were proposed to estimate wake-up time of devices. Analysis model was built by probability theory and matrix way, which could monitor different communication scenarios. However, these algorithms have not embodied the RAW communication mechanism. In [11], low collision probability was achieved through access control to limit the number of devices contending by setting the threshold of access point (AP), and making a decision by comparing threshold with the numbers that devices generate in the network. It only fitted for authentication stage instead of transmitting and receiving process. In [12], the RAW mechanism is analyzed based on non-cross slot boundary holding scheme. However, it focuses on the holding scheme instead of RAW size and retransmission. Our previous work [13] proposed adaptive RAW for IEEE 802.11ah based networks. The aim is to achieve highest uplink energy efficiency by estimate optimal combination of the number of time slots and proper group size involved for one uplink RAW. However, we 
have not jointly considered the energy efficiency and delay in novel retransmission scheme.

The main contribution of these papers is to improve energy efficiency based on transmission probability and reducing collision for IEEE 802.11ah. Less work has been done to optimise the energy efficiency and delay with joint consideration of RAW size and retransimission scheme based on clustering size. To address the above joint consideration, in this paper we study an optimisation problem aiming at maximizing uplink energy efficiency and reducing delay through RAW control. An energy-delay aware window algorithm is proposed to determine the number of time slots and internal slot interval in one RAW for various group scales based on novel retransmission scheme for uplink communications. An optimal solution is derived by applying Gradient Descent approach, an efficient way for the global optimization problem.

The remainder of the paper is organised as follow. Section II describes system model about IEEE 802.11ah MAC protocol. Problem formulation is given in Section III. Section IV presents the derivation of optimal solution. Section $\mathrm{V}$ provides the simulation results to show the efficacy of algorithm we proposed. Finally, section VI concludes the paper.

\section{SYSTEM MODEL}

We consider a single-hop topology for dense IEEE 802.11ah IoT networks as a single Access Point (AP) with a high number of devices. RAW groups the devices and splits the channel into equal time slots [14]. All devices in this network listen in the beginning of beacon frame called Target Beacon Transmission Time (TBTT) to obtain scheduling information that indicates which RAW they are allocated to. Then devices would fall into sleep mode until turning to their RAW to attempt accessing. For each RAW, there are $M$ time slots and $N$ devices limited by lowest and highest Associated Identifier (AID) of devices which indicat the location, traffic, type, energy saving mode etc [6].

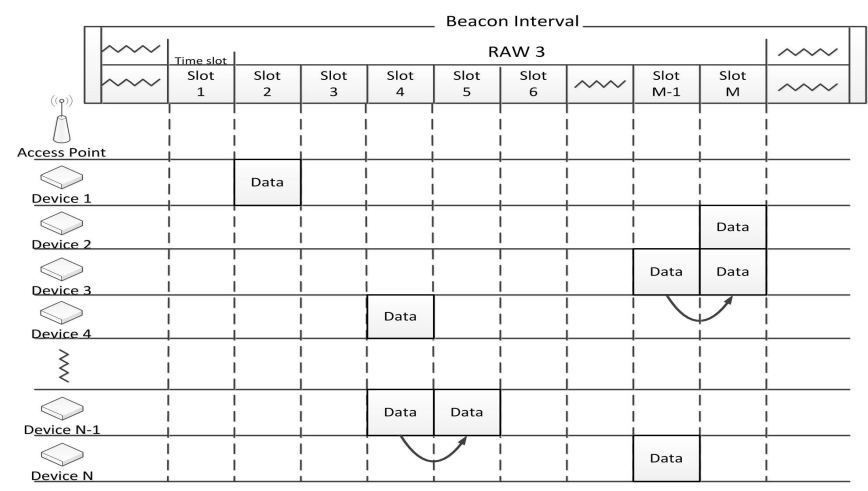

Fig. 1. Operation of RAW

In IEEE 802.11ah uplink communications, the devices who have buffered data for the AP randomly select a time slot in allocated RAW and attempt to access the channel as shown in Fig. 1. If there is only one device in a slot, it could access directly as Device 1 transmits packet in Slot 2 directly without contention. When there are more than one device choosing the same time slot, for example, Device 3, Device N in Slot $\mathrm{M}-1$, and the same for Device 4 and Device N-1 in Slot 4, they would come into the back-off stage to avoid collision by doubling contention window and trying again until reaching the slot boundary. If accessing the channel successfully, device requests uplink communication by sending PowerSave-poll (PS-poll) message to the AP. AP responses with an ACK to confirm connection. After the first handshake, the device transmits buffered data frame and waits for ACK from AP [15]. We assume if not accessing the channel, it would retransmit in the next slot, for example, when Device $\mathrm{N}$ has accessed the channel in Slot M-1, Device 3 retransmits in Slot M, which needs to contend with Device 2, and Device 4 retransmits in Slot 5 as Device 4 has accessed in Slot 4. But when they can not retransmit successfully in the following slot, they need to retransmit in subsequent allocated RAW. The process is repeated, one RAW by one RAW, until the end of beacon frame.

\section{PROBLEM FORMULATION}

In this section, we formulate energy efficiency and delay for IEEE 802.11ah uplink communications. The process of devices to access channel in IEEE 802.11ah is formulated by Markov Chain and the energy efficiency and delay are deried.

For the uplink communication in MAC layer of IEEE 802.11ah, the number of devices to contend is limited by RAW. Time slots in RAW are selected by devices randomly. Since random selection, the states of each time slot are different, and retransmission makes it depend on the state of previous slot, so Markov Chain is applied to monitor accessing process of each time slot in one RAW.

The process in Markov Chain is represented by three parameters $j, n_{j}, s_{j}$, where $j$ is the index of time slot in one RAW; $n_{j}$ shows there are $n_{j}$ devices to choose the $j t h$ time slot; $s_{j}$ is the number of devices accessing channel successfully, which is illustrated as Fig. 2.

The transition probability of adjacent states could be donated as Equation 1, where $P_{i j}$ is the probability to transfer from slot $i$ to slot $j ; \mathrm{P}_{d_{-} n_{j}}$ is the probality of there are $n_{j}$ to choose the same time slot; $\mathrm{P}_{s_{-} n_{j}}$ is the probality of successfully accessing.

As for a time slot in RAW, the probability that there are $n_{k}$ devices to choose is

$$
P_{d_{-} n_{k}}=\left(\begin{array}{c}
N_{u l} \\
n_{k}
\end{array}\right)\left(\frac{1}{M_{u l}}\right)^{n_{k}}\left(1-\frac{1}{M_{u l}}\right)^{N_{u l}-n_{k}},
$$

where $M_{u l}$ is the number of uplink time slots contained in one RAW; $N_{u l}$ is the number of devices that could be involved in one RAW intending to access channel; $n_{k}$ is the number of devices to choose the $k t h$ time slot.

According to [7], there are two cases for a device to transmit uplink packet successfully in one slot.

- Case 1: a time slot chosen by only one device. This device would transmit a packet successfully without other contending devices. 


$$
P_{i j}= \begin{cases}\mathrm{P}_{d_{-} n_{j}} \mathrm{P}_{s_{-} n_{j}}, & \text { if }\left(i=0,0<n_{j}<N_{u l}, s_{j}=1\right) \\ \mathrm{P}_{d_{-} n_{j}}\left(1-\mathrm{P}_{s_{-} n_{j}}\right), & \text { if }\left(i=0,0<n_{j}<N_{u l}, s_{j}=0\right) \\ \mathrm{P}_{d_{-} n_{j}} \mathrm{P}_{s_{-}\left(n_{j}+n_{i}-s_{i}\right)}, & \text { if }\left(i>0,0<n_{j}<\left(N_{u l}-\sum_{\substack{i-1 \\ k=0 \\ i-1}} n_{k}\right), s_{j}=1\right) \\ \mathrm{P}_{d_{-} n_{j}}\left(1-\mathrm{P}_{s_{-}\left(n_{j}+n_{i}-s_{i}\right)}\right), & \text { if }\left(i>0,0<n_{j}<\left(N_{u l}-\sum_{k=0} n_{k}\right), s_{j}=0\right) \\ 0, & \text { otherwise }\end{cases}
$$

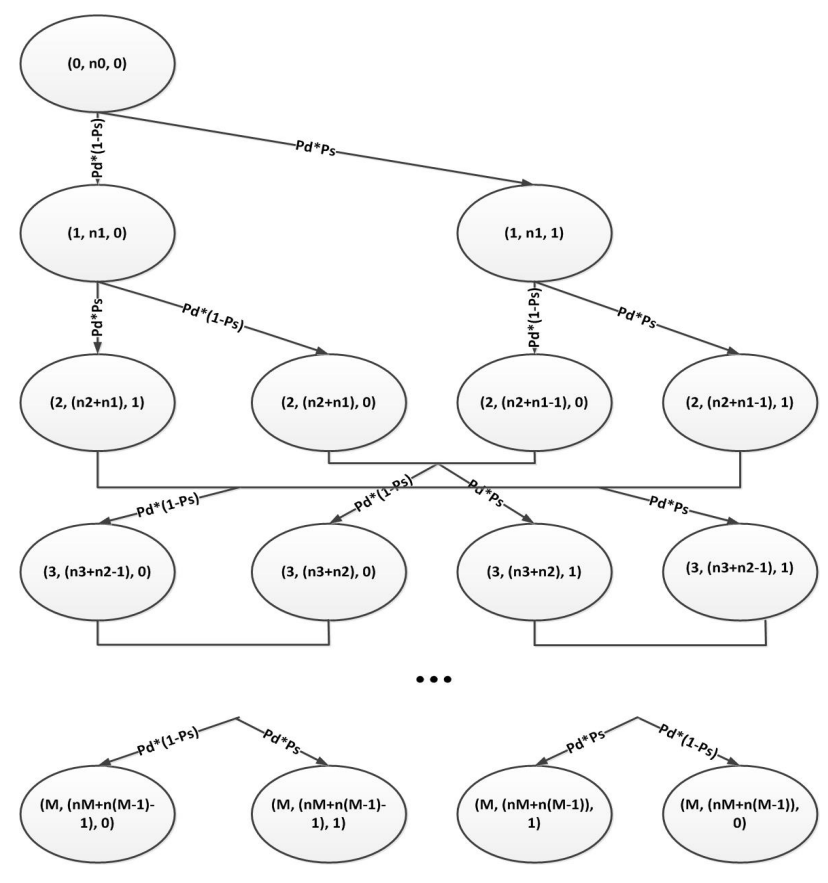

Fig. 2. Markov Chain.

- Case 2: a time slot chosen by multiple devices. The devices will go into back-off stage due to contention, and one of them will succeeds in accessing the channel at the first back-off stage.

So the successful accessing probability could be described as

$$
P_{s_{-} n_{k}}=\left\{\begin{array}{l}
1, \text { if }\left(n_{k}=1\right) \\
P_{s_{-} n_{k_{-}} \text {backoff }}, \text { if }\left(\mathrm{n}_{k}>1\right)
\end{array},\right.
$$

where $n_{k}=1$ denotes the situation as the Case $1 ;\left(\mathrm{n}_{k}>1\right)$ is related to Case $2 ; P_{s_{-} n_{k_{-}} \text {backoff } f}$ is the probability as Case 2 to access channel in first back-off stage.

For the Case 2, in view of $(i-1)$ other contending devices, only one device will success in accessing the channel at the first back-off. The probability of a minimum contention window as the first back-off stage is:

$P_{s_{-} n_{k} \_ \text {backoff }}=$ where $W_{\min }$ is the minimal size of contention window.

The total number of packets could be sent in one RAW is

$$
\sum_{0}^{M_{u l}} s_{k}=\sum_{0}^{M_{u l}} s_{k \_1}+\sum_{0}^{M_{u l}} s_{k \_2},
$$

where $\sum_{0}^{M_{u l}} s_{k_{-}}$is the number of packets sent as Case 1; $\sum_{0}^{M_{u l}} s_{k_{-} 2}$ is the number of packets sent as Case 2.

The transmission energy consumption of one RAW is

$$
\begin{aligned}
E_{t \_u l}= & E_{t 1 \_u l} \sum_{0}^{M_{u l}} s_{k_{-} 1}+E_{t 2 \_u l} \sum_{0}^{M_{u l}} s_{k_{-} 2} \\
& +E_{t 3 \_u l}\left(N_{u l}-\sum_{0}^{M_{u l}} s_{k}\right)+E_{c o n} M_{u l} \tau_{u l} N_{u l},
\end{aligned}
$$

where $E_{t 1 \_u l}$ is the energy consumption when transmitting a packet successfully as Case $1 ; E_{t 2 \_u l}$ is the energy consumption when transmitting a packet successfully as Case $2 ; E_{t 3 \_u l}$ is the energy waste when there is collision both in its slot and next slot so that it needs to retransmit in another RAW; $E_{\text {con }}$ is the contention power in one RAW, which is the energy consumed when it is in wake-up mode; and $\tau_{u l}$ is the time duration of one time slot.

The size of RAW also determines the energy consumption of transmitting overhead information, because when the window duration is too small, the overhead information of each device would be high due to the scheduling information that needs to be transmitted multiple times in a short time. And if the number of devices involved in one RAW is small, it also needs massive scheduling information to realize network communication. So the energy consumption of head information is related to $N_{u l}$ and $M_{u l}$ :

$$
E_{\text {head_ul }}=\frac{\alpha}{M_{u l}} \times \frac{\beta}{N_{u l}},
$$

where $\alpha$ is the parameter indicating traffic and $\beta$ is the parameter related to overall number of devices in the scenario.

Energy efficiency of one RAW could be evaluated by the data rate it provides and overall energy consumption. Data rate could be formulated as

$\sum_{k=0}^{W_{\min }-1}\left\{\prod_{0}^{k}\left[1-\frac{1}{W_{\min }}\left(1-\frac{k}{W_{\min }}\right)^{n_{k}-1}\right]\right\} \frac{1}{W_{\min }}\left(1-\frac{k+1}{W_{\min }}\right)^{n_{k}-1}$,

$$
R_{u l}=\frac{\sum_{0}^{M_{u l}} s_{k} \times \gamma_{u l}}{\tau_{u l} M_{u l}}
$$


where $\gamma_{u l}$ is the packet size. $\sum_{0}^{M_{u l}} s_{k} \times \gamma_{u l}$ is the total length of packets could be transmitted for $N_{u l}$ devices in $M_{u l}$ time slots. $\tau_{u l} M_{u l}$ is the total time of one RAW.

The overall energy consumption consists of transition power and overhead power when $N_{u l}$ devices attempt to communicate with AP during one RAW, which could be denoted by

$$
E_{\text {overall_ul }}=E_{t \_u l}+N_{u l} E_{\text {head_ul }} \text {. }
$$

Thus energy efficiency is

$$
E E_{u l}=\frac{R_{u l}}{E_{\text {overall }}}=\frac{\gamma_{u l} \sum_{0}^{M_{u l}} s_{k}}{\tau_{u l} M_{u l}\left(E_{t_{-} u l}+N_{u l} E_{\text {head_ul }}\right)} .
$$

The average delay for all the packets transmitted in one RAW is

$$
D_{u l}=\frac{\sum_{0}^{M_{u l}} k \tau_{u l} s_{k}}{\sum_{0}^{M_{u l}} s_{k}}
$$

So the energy efficiency could be denoted as

$$
E E_{u l}=\frac{R_{u l}}{E_{\text {overall }}}=\frac{\gamma_{u l} \sum_{0}^{M_{u l}} k \tau_{u l} s_{k}}{\tau_{u l} M_{u l} D_{u l}\left(E_{t \_u l}+N_{u l} E_{h e a d \_u l}\right)} .
$$

In order to estimate number of packets transmitted successfully, the average numbers of packets that could be transmitted successfully are formulated as Equation 10.

Thus energy efficiency and delay is a function related to the number of devices involved $\left(N_{u l}\right)$ and time slot in one RAW $\left(M_{u l}, \tau_{u l}\right)$. We could jointly maximize energy efficiency and delay by finding optimal $M_{u l}$ and $\tau_{u l}$ based on diverse groups.

\section{ENERGY EFFICIENT RAW}

In this section, the energy efficient RAW is devied based on the novel retransmission scheme.

Let $\xi=\sum_{0}^{M_{u l}} s_{k}$ and $E_{t_{-} a v g}$ be the average energy consumption to send a packet successfully in a slot, the energy efficiency from Section III can be simplified as

$E E_{u l}=$

$\frac{\gamma_{u l} \xi \tau_{u l}-1}{M_{u l} E_{t \_a v g \_u l} \xi+M_{u l} E_{t 3 \_u l}\left(N_{u l}-\xi\right)+E_{c o n} M_{u l} \tau_{u l} N_{u l}+\alpha \beta}$.

$\xi$ is a function related to $P_{d_{-} n_{k}}$ and $P_{s_{-} n_{k}}$ which can be denoted as $\xi=\sum P_{d_{-} n_{k}} \times P_{s_{-} n_{k}}$, a monotone increasing function along the increment of the number of time slots, because the amount of packets that could be sent increases with increament of the RAW size. For denominator, $M_{u l} E_{t \_a v g \_u l} \xi+M_{u l} E_{t 3 \_u l}\left(N_{u l}-\xi\right)$ is a decreasing function, since $\bar{\xi}$ increase with enlarging RAW duration and $E_{t 3 \_u l} \geq$ $E_{t \_a v g \_u l}$. And $E_{c o n} M_{u l} \tau_{u l} N_{u l}$ is a increasing function for $M_{u l}$, thus denominator is a convex function and the $E E_{u l}$ is concave with a maximum value.

In order to guarantee the transmission, the time slots could be determined by device type. Different packet sizes relate to adptive time slots respectively, which could be described as

$$
\tau_{u l} \geq \frac{\gamma_{u l}}{v}+\tau_{\text {back_off }},
$$

where $v$ is the transmission rate of IEEE 802.11ah and $\tau_{\text {back_off }}$ is the back-off time when more than one devices to choose the same time slot.

We find optimal RAW duration for different group scales based on novel retransmission scheme to maximize uplink energy efficiency and decrease delay by applying Gradient Descent, a fast algorithm to find the optimum of large search space as shown in Algorithm 1.

$\overline{\text { Algorithm } 1 \text { Gradient Descent Energy-Delay Aware RAW }}$ Control with Novel Retransmission Algorithm

1: Step 1: AP detects the devices type and the number of devices in the group.

2: Step 2: The uplink time slot durations are set based on the type of devices, which are the constrants.

3: uplink time slot duration $\geq$ transmit time + back_off time;

4: Step 3: The uplink window size is determined according to the number of uplink devices.

5: Step 3.1: estimate optimal uplink RAW size for a certain group.

6: loop

7: $\quad$ Initialize $M_{\text {old }}$ and $M_{\text {new }}$ as two random numbers.

8: $\quad$ EE_derivative $(M)=\operatorname{Diff}\{-E E(M, N), M\}$

9: $\quad$ while $a b s\left\{E E\left(M_{\text {old }}\right)-E E\left(M_{\text {new }}\right)\right\} \geq$ precision do

10: $\quad \partial=0.01$

11: $\quad M_{\text {old }}=M_{\text {new }}$

12: $\quad M_{\text {new }}=M_{\text {old }}-\partial \times E E_{\text {derivative }}(M)$

13: end while

14: $\quad$ return $M$

15: end loop

16: Step 3.2: Set RAW as $M$ time slots for this group. The devices fall into sleep mode and wake up when coming to their allocated window.

17: Step 3.3: $N$ devices randomly select one of $M$ time slots in RAW and attempt to do uplink communications with AP.

18: $N$ devices in the group choose one of $M$ slots randomly.

19: Devices attempt to access channel to establish connection with AP as Case 1 and Case 2.

20: When the device could not access in the its slot, re-attempt in the following slot. The operation is same as in the previous one.

21: If the device still could not access in next slot, the device needs to waite for next allocated RAW to re-access.

This algorithm is a standard Gradient Descent approach to find the optimal solution. It starts with an arbitrary value based on a random value, and then compare the existing optimum with a new function results with input that adding a gradient value. When upcoming one performs better, accept new one, otherwise, reserve previous. Step by step to find the optimal value according to gradient descent route. 


$$
\begin{aligned}
& s_{1}=\sum_{n_{1}=1}^{N_{u l}} P_{d \_n} P_{s_{-} n_{1}} \\
& s_{2}=\sum_{n_{2}=1}^{N_{u l}-n_{1}} \sum_{n_{1}=1}^{N_{u l}}[\underbrace{P_{d_{-} n_{1}}\left(1-P_{s_{-} n_{1}}\right) P_{d_{-} n_{2}}}_{\varepsilon_{21}} P_{s_{-}}\left(n_{2}+n_{1}\right)+\underbrace{P_{d_{-} n_{1}} P_{s_{-} n_{1}} P_{d_{-} n_{2}}}_{\varepsilon_{22}} P_{s_{-}}\left(n_{2}+n_{1}-1\right)] \\
& s_{3}=\sum_{n_{3}=1}^{N_{u l}-n_{1}-n_{2}} \sum_{n_{2}=1}^{N_{u l}-n_{1}} \sum_{n_{1}=1}^{N_{u l} l}[\underbrace{\left(\varepsilon_{21}\left(1-P_{s_{-}\left(n_{3}+n_{2}\right)}\right)+\varepsilon_{22}(1-\underbrace{\left.\left(\varepsilon_{21} P_{s_{-}\left(n_{3}+n_{2}\right)}+\varepsilon_{s_{-}}\left(n_{3}+n_{2}-1\right)\right)\right) P_{d_{-} n_{3}}}_{\varepsilon_{32}} P_{s_{-}\left(n_{3}+n_{2}-1\right)}) P_{d_{-} n_{3}}+n_{2}\right)}_{\varepsilon_{31}} P_{s_{-}\left(n_{3}+n_{2}-1\right)}] \\
& \text {... } \\
& \begin{array}{r}
s_{j}=\sum_{n_{j}=1}^{N_{u l}-\sum_{k=1}^{j-1} n_{k}} \sum_{n_{(j-1)}=1}^{N_{u l}-\sum_{k=1}^{j-2} n_{k}} \ldots \sum_{n_{1}=1}^{N_{u l}}[\underbrace{\left(\varepsilon_{(j-1) 1}\left(1-P_{s_{-}\left(n_{j}+n_{(j-1)}\right)}\right)+\varepsilon_{(j-1) 2}\left(1-P_{s_{-}\left(n_{j}+n_{(j-1)}-1\right)}\right)\right) P_{d_{-} n_{j}}}_{\varepsilon_{j 1}} P_{s_{-}\left(n_{j}+n_{(j-1)}\right)}) \\
+\underbrace{\left(\varepsilon_{(j-1) 1} P_{s_{-}\left(n_{j}+n_{(j-1)}\right)}+\varepsilon_{(j-1) 2} P_{s_{-}\left(n_{j}+n_{(j-1)}-1\right)}\right) P_{d_{-} n_{j}}}_{\varepsilon_{j 2}} P_{s_{-}\left(n_{j}+n_{(j-1)}-1\right)}]
\end{array}
\end{aligned}
$$

\section{SimUlation RESUlt AND ANALYSIS}

In this section, the performance of optimization algorithm for RAW is evaluated in Matlab. We consider a one-hop topology as describe in the system model and apply the energy-delay aware RAW control to the AP and the novel retransmission scheme to collised devices.

We apply wake-up/sleep mode for all devices. At each RAW, there are $N_{u l}$ devices being allocated. When coming to their RAW, devices keep wake-up mode and randomly select their own time slot to do transmission to the AP, otherwise they would fall into sleep mode. We assume that every device involved has exactly one packet to transmit for uplink communications during one RAW. The main simulation parameters are given in Table I.

TABLE I

SIMULATION PARAMETERS

\begin{tabular}{|c|c|c|c|}
\hline Parameter & Value & Parameter & Value \\
\hline frequency & $0.9 \mathrm{GHz}$ & data rate & $100 \mathrm{kbps}$ \\
\hline transmit power for Case1 & $1.346 \mathrm{mw}$ & transmit power for Case2 & $2.5 \mathrm{mw}$ \\
\hline collision power & $3.0 \mathrm{mw}$ & idle listen power & $0.001 \mathrm{mw}$ \\
\hline min contention window & 8 & max contention window & 1024 \\
\hline$\alpha$ & 200 & $\beta$ & 200 \\
\hline
\end{tabular}

The uplink energy efficiency varying over diverse number of devices per group based on two different packet sizes (512 bits and 1024 bits) is shown in Fig. 3. The proposed RAW is to set the best matched window size and internal slot interval based on group to achieve optimal energy efficiency with the novel retransmission scheme as the the collised devices retransmit in next slot. The exsiting RAW is to set the adaptive number of slots based on optimization of energy efficiency and the retransmission is in another allocated RAW when there is a collision [13]. It can be observed that the proposed RAW perfoms better than the existing one with $113.3 \%$ and $63.3 \%$

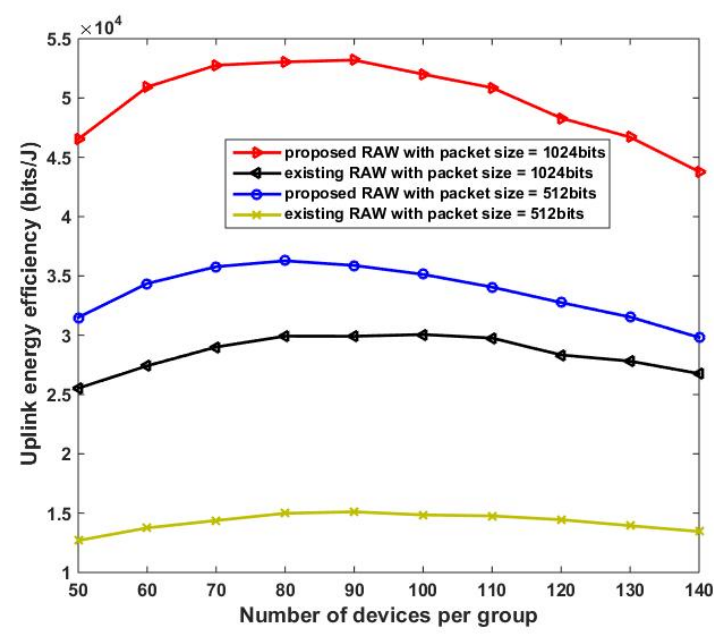

Fig. 3. Uplink energy efficiency comparison with various amount of devices.

improvement for packet size $=512$ and 1024 bits repectively

For random access in uplink communication, there are many empty slots without device to choose. The proposed RAW with retransmission scheme is to retransmit in next slot for collised devices in crowded slot (selected by more than one device), which could reuse the empty slots, so there are more packets could be transmitted in proposed RAW, leading to high energy efficiency. The adaptive time slots could decrease the wake-up power, which is a way to reduce the energy consumption, thus the proposed RAW could outperform than the existing RAW.

When the number of devices is low, the header energy consumption would be high to achieve whole network communications, since scheduling information need to send mutiple times after a short time. So with the increment of group scale, the header energy consumption would decrese, which is a dominate factor to increase energy efficiency. However, if 
the number of devices per group is too large, the collision probability would be high which leads to consume more energy and low data rate, two parameters for low efficiency, thus the trend of curves will go down. Since the data rate increases with the increment of packet size based on the same number of devices, the energy efficiency of 1024 bits per packet is higher than 512 bits.

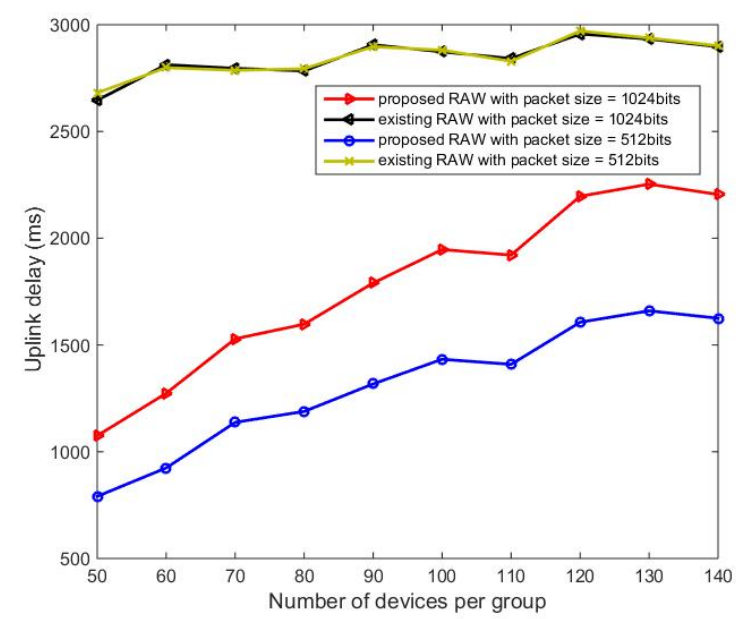

Fig. 4. Uplink delay comparison with various number of devices.

Fig. 4 shows the uplink delay with two packet sizes as it varies along diverse number of devices. The proposed RAW reduces $53.4 \%$ and $37.9 \%$ in uplink delay for packet size $=512$ bits and 1024 bits repectively. The novel retransimission scheme could lead to more packet transmitted in one RAW so that the average delay for one RAW is lower than the existing one. The adaptive slot interval could alleviate the time waste, which is the other reason for decreasing the delay.

The delay of existing RAW for both two packet sizes are same due to the same duration and number of time slots. Since the transmision time increases with the packet size in our proposed control algorithm, the average delay of 1024 bits is higher than the one of 512 bits. And more number of devices will lead to longer duration of RAW in order to avoid collision, thus the average delay would increase along the increment of the number of devices. However, sometimes the increasing rate of the number of time slots is lower than the increasing rate of devices, so the curve is not smooth such as the number of devices changing fron 100 to 110 .

Simulation results demonstrate the propoesd RAW control scheme could bring about superior energy efficiency and delay for IEEE 802.11ah uplink communications.

\section{CONCLUSiOnS}

In this paper, an energy-delay aware access window with retransmission control algorithm is proposed for IEEE 802.11ah networks to jointly optimize uplink communications energy efficiency and delay through adapting RAW duration and internal slot interval for different group size with diverse number of devices in one group to access simultaneously.
And the retransmission scheme could reuse the empty slots due to random selection of devices. The algorithm is built based on Markov Chain and probability theory to evaluate transmission probabilities of various states a device may fall into when sending a packet during one RAW. On account of that, overall energy consumption and data rate are estimated to contribute to energy efficiency. The optimal solution is derived by applying Gradient Descent approach. Simulation results demonstrate that the joint the optimal number of time slots and internal slot interval in one RAW based on retransmission scheme could lead to improvement of energy efficiency and decrease of delay for one RAW.

\section{REFERENCES}

[1] S. Aust, R. Prasad, I.G.M.M. Niemegeers, "IEEE 802.11ah: Advantages in standards and further challenges for sub $1 \mathrm{GHz}$ Wi-Fi," 2012 IEEE International Conference on Communications (ICC), 2012, pp. 68856889.

[2] IEEE Standard for Information Technology Telecommunications and information exchange between systems Local and metropolitan area networks Specific requirements Part 11: Wireless LAN Medium Access Control (MAC) and Physical Layer(PHY) specifications Amendment 10: Mesh Networking, 2011.

[3] Wi-Fi Alliance. (2016). Wi-Fi Alliance introduces low power, long range Wi-Fi HaLow [Online]. Available: https://www.wi-fi.org/newsevents/newsroom/wi-fi-alliance-introduces-low-power-long-range-wi-fihalow.

[4] A. Hazmi, J. Rinne, M. Valkama, Feasibility study of IEEE 802.11ah radio technology for IoT and M2M use cases, 2012 IEEE Globecom Workshops (GC Wkshps), IEEE, 2012, pp. 1687-1692.

[5] Y. Seok. (2013). Backoff Procedure in RAW [Online]. Available: http://mentor.ieee.org/802.11/dcn/13/11-13-0080-00-00ah-backoffprocedure-in-raw.ppt.

[6] E. Khorov, A. Lyakhov, A. Khorov, A. Guschin, A survey on IEEE 802.11ah: An enabling networking technology for smart cities, Computer Communications (2014)

[7] C.W. Park, D. Hwang, T. Lee, Enhancement of IEEE 802.11ah MAC for M2M Communications. Communications Letters, July, 2014, pp. 11511154.

[8] F. Vazquez-Gallego, M. Rietti, J. Bas, J. Alonso-Zarate, L. Alonso, Performance Evaluation of Frame Slotted-ALOHA with Succesive Interference Cancellation in Machine-to-Machine Networks. European Wireless 2014, pp. 403-408.

[9] R.P. Liu, G.J. Sutton, I.B. Collings, Power save with offset listen interval for IEEE 802.11ah smart grid communications, 2013 IEEE International Conference on Communications (ICC), IEEE, 2013, pp. 4488-4492.

[10] R.P. Liu, G.J. Sutton, I.B. Collings, WLAN power save with offset listen interval for machine to machine communications, Wireless Communications vol.13 No.5.

[11] Y. Zhou, H. Wang, S. Zheng, Z.Z. Lei, Advances in IEEE 802.11ah standardization for machine-type communications in sub-1GHz WLAN, 2013 IEEE International Conference on Communications Workshops (ICC), IEEE, 2013, pp. 1269-1273.

[12] M. Qutab-ud-din, A. Hazmi, B. Badihi, A. Larmo, J. Torsner and M. Valkama, Performance analysis of IoT-enabling IEEE 802.11 ah technology and its RAW mechanism with non-cross slot boundary holding schemes. 2015 IEEE 16th International Symposium on a World of Wireless, Mobile and Multimedia Networks (WoWMoM), IEEE, 2015, pp. 1-6.

[13] Y. Wang, Y. Li, K. K. Chai, Y. Chen, J. Schormans, Energy-aware Adaptive Restricted Access Window for IEEE 802.11ah Based Networks, 2015 IEEE 26th annual International Symposium on Personal, Indoor and Mobile Radio Communications (PIMRC), IEEE, 2015, pp. 12531257.

[14] J. Kim. (2013). RAW Assignment follow up [Online]. Available: http://mentor.ieee.org/802.11/dcn/13/11-13-0510-01-00ah-rawassignment-follow-up.ppt.

[15] Specification Framework for TGah, IEEE Std. 802.11-11 1137r15, May 2013. 